\title{
A Framework to Support the Design of Digital Initiatives in Social Science Based Research
}

\author{
Stuart Moran, Sophie Berckhan and Alison Clarke \\ Digital Research Team, University of Nottingham, Nottingham, UK \\ \{stuart.moran, sophie.berckhan, alison.clarke\} @nottingham.ac.uk
}

\begin{abstract}
The rapid development of new digital technologies has increased the expectations of academic research outputs. To meet these pressures, researchers have greater expectations of their digital work environments. In this paper we outline the drivers for digital transformation at our institution and how this manifested itself in a series of exploratory projects called digital initiatives. Based on our findings we propose a Digital Research Initiative framework to support research institutions in assessing and implementing digital transformation.
\end{abstract}

Keywords: Academic Support, Digital Research, Digital Transformation

\section{Introduction}

For the past decades, academic institutions have undergone rapid change driven by globalisation, greater social mobility, new funding patterns and the introduction of new technologies [1]. This has led to a change in the way institutions operate, moving away from a self-centred to a self-determined model and environment [2]. More recently, several new trends have emerged relating to the scale at which research is undertaken, the need to evidence the public benefit of research and a changing set of government and funder regulatory requirements. This has placed new pressures on researchers to deliver high-quality results, at a fast pace, with real-world relevance and in compliance with regulations. Consequently, researchers have ever increasing expectations of their institution to offer the appropriate tools and environment that enable them to respond to these pressures. This is perhaps most prominent in terms of the Information Technology (IT) provision, with researchers expecting timely access to the very latest and ever changing digital tools and services. This has created new opportunities for institutional IT services to make a shift from being a purely operational function, toward a strategic enabler and leader. This role change will result in digital services that not only continue to support research but also actively inspire new research possibilities.

In this paper we seek to outline the formation of our institution's digital research vision and explore how this manifested itself through a series of small-scale digital transformation projects specific to Social Sciences. Based on our findings we then propose a framework that can support institutions in assessing their readiness for digital transformation and broadly inform decision making on the types of solution to implement. 


\section{The Need for Digital Transformation}

Prior to exploring our institution's digital transformation vision, we must first understand more clearly the broader research context and drivers of change:

Transparency and Compliance. Research councils and funders are placing greater emphasis on the need for research transparency and open access to research findings. This will also include in the requirement that research data be suitably archived and preserved for the long term [3]. In terms of the management of data, the General Data Protection Regulation (GDPR) also places new constraints on the way personally identifiable research data is acquired and managed [4]. Cybersecurity is also a major consideration particularly in light of sensitive research data topics, commercial partners and patentable research outputs.

Collaboration and Skills. A stronger emphasis is now placed on large scale critical mass research driven, amongst others, by the Global Challenges Research Fund [5]. These are often addressed by multi-disciplinary and multi-institutional consortia driving the need to work collaboratively on larger projects. To be competitive research groups must continually refine their areas of expertise and promote their ability to add value in interdisciplinary projects more clearly. Interestingly, this behaviour means that while researchers are actively exposed to new techniques and knowledge from other disciplines, they do not necessarily need to apply and acquire those skills for themselves. This puts groups with specialist skills in high-demand [6], creating bottlenecks in interdisciplinary research

Digital Equipment and Data. Recent developments in precision manufacturing and technology development have made numerous highly specialised instruments available to researchers [7]. The high cost of these equipment and services means effective resource sharing is required. This has led to the consolidation of research equipment into large centres and shared regional and national facilities. The Wakeham Review [8] concluded that shared resources and services will provide opportunities for better science. However, as instruments can generate significant volumes of data, their centralisation has created new challenges about the speed and safety of moving this data between institutions. Furthermore, the amalgamation of equipment has also further consolidated the availability of expertise [9].

Impact and Metrics. With public funds used to support research, there is a growing need to demonstrate the public benefit of research outputs. These developments have led to a greater emphasis on research metrics with a focus on the production of outputs with the highest possible influence. The presentation of research outcomes to the public has created opportunities for recognition outside of academic disciplines through a range of media channels [10]. 
Underpinning Digital Capabilities. Institutions are in an intriguing situation where digital technologies and services both serve as the cause of the changing environment but also as the route to a viable solution. Research funders have an expectation that institutions will provide researchers with a "well-founded laboratory" that includes IT infrastructure and services. Many institutions are in a position where the digital demand and expectations of researchers currently outweighs their ability to offer the capabilities. Without a step-change in the digital provision, institutions risk not being able to serve what is becoming a basic research need and will fail to support researchers in fulfilling their research ambitions. This would in turn reduce an institutions research income, power and reputation.

\section{$3 \quad$ Informing the Digital Research Strategy}

In response to the changing work environment of researchers, our institution took steps toward creating a research environment that not only supports researchers' current needs, but also actively encourages them to increase the scope of their ambitions. A digital research vision was formed that recognized that the existing digital service provision was no longer viable, and that there was an opportunity for the IT function to not only enable, but to actively create innovative research. The vision outlined a research environment that would not only facilitate high quality research, but ultimately change the culture and working practices of researchers. While at a high level, the areas that needed investment were broadly known, they were ill-defined and poorly understood. This is in part due to communication and knowledge barriers between IT services and the highly specific and complex research areas across the institution.

Without a means of bridging this gap, the institution was not in a position to deliver an appropriate research environment. In response, a small innovative and strategic Digital Research Team was established within IS, made up of specialist post-doctoral researchers each with a portfolio of ambitious digital research projects. The collective research experience of the team covered all five faculties of the institution. It was anticipated that this unique combination of research knowledge and experience would allow researchers' needs to be more effectively captured and articulated to the IT delivery teams. Furthermore, these roles would provide the expertise to challenge and inspire researchers with new digital research opportunities. A survey of Russel Group universities revealed a focus on digital humanities centres, academic-led digital innovation centres, or specific roles such as research software engineers, data managers and data scientists. There were no indications that a similar institution-wide strategic digital research team is currently in place at these institutions. In order to establish the specific research needs of the institution, researchers across each faculty were approached by the Digital Research Team. The remainder of this papers focuses on the findings of the Faculty of Social Sciences as due to the broad range of disciplines it suitably represents the challenges faced across the institution. 


\subsection{Digital Research Environment in Social Science}

At our institution Social Science is an exceptionally broad field, with a range of research methods applied across different disciplines in different contexts. In order to better understand the 'digital-readiness' of the faculty, approximately 100 researchers were met with over a 6-month period. This was on a one-to-one basis and intended to establish (1) the types of research methods adopted (2) the digital barriers faced by researchers and (3) an outline of the long-term research ambitions. Following these discussions, three key themes emerged around digital skills, digital systems and digital support.

Digital Skills in Research. As research technologies have advanced over the last 1520 years, a digital divide has emerged, with those who make use of technologies to their fullest, and those who miss opportunities. Particularly in the qualitative space, there appears to be either a misunderstanding as to the broad capabilities of current technologies, or a perception that technology cannot support their work. For those who engage in quantitative work, there are many challenges around selecting the most computationally efficient technique or hardware platforms to use for a given dataset. Where programming is required, researchers are generally self-taught and often viewed their code and statistical modules as inefficient. This was often cited as the reason for not sharing these research outputs more widely. Without a complete understanding of the research possibilities with the latest digital tools, techniques and technologies, researchers are missing opportunities to do more with their research with fewer resources. However, it is the barriers to entry, not the ambition, that frequently outweigh the digital opportunities available.

Digital Systems in Research. There is a large variety of data being created and captured by researchers, both in terms of their file formats and the degree of sensitivity of their content. Researchers are offered many different storage solutions by our institution considering their data requirements. However, a consequence of being able to satisfy this range of needs is that identifying the appropriate solution is difficult, creating barriers for researchers. Furthermore, in order to practically and safely share research data with the community, researchers are required to adhere to a cumbersome controlled access process, which acts as a barrier to sharing data. In some extreme instances, researchers have chosen not to pursue lines of enquiry due to the inefficient and uncertain storage mechanisms. Computationally intensive research is often completed using 'always-on' local desktop machines, running for days at a time. This is because the high performance computer at our institution not only requires a set level of expertise to access and utilize, but is also limited exclusively to the use of the Linux operating system. There are also a range of digital devices that can support the capture of research data that are underutilized.

Research findings and opportunities are missed as a consequence of data not being stored or data being unusable due to poor management. Similarly, without the appropriate compute facilities, key insights into large data sources continue to be difficult to elicit or simply unachievable. 
Digital Support in Research. Researchers are broadly unaware of the types of professional support that is already available to them, in areas such as social media, digital research, data management and intellectual property. Some support services are run by researchers for researchers but gaining timely access to these can be difficult due to limited resources. There are also no current services available to help develop small scale code pilots or advise on specific statistical technologies. While the institution does provide state-of-the-art computational facilities and guidance on its use, there is a perception that this is tailored toward expert users. Without support and awareness of the use of new research technologies and techniques, researchers are unlikely to adopt them, resulting in missed opportunities and less competitive grant applications.

\section{$4 \quad$ Digital Research Initiatives}

Following the elicitation of researcher needs across the institution, three key strategic areas for investment were identified: Research Data Management, Compute and Analytics and Communication and Collaboration. The aim was to introduce new digital technologies and services that support researchers in each of these key areas which support and challenge the working practices and culture of research. In order to explore what digital technologies and services are likely to be most successful for researchers, a small scale rapid-delivery approach was adopted. These projects, or digital research initiatives (DRIs), seek to introduce and explore innovative research methods, off-theshelf and custom technologies, and specialist knowledge. The DRI's are essentially meta-research projects with the goal of exposing researchers to new ways of working and technologies, and directly demonstrating their value. In order to secure buy-in from researchers, the DRI's were designed to align with existing practices where possible, and produce research outputs such as publications and grant applications. The outcomes of the projects were then used as case studies to inspire the wider research community to engage in new ways of working. In the next section we discuss some of the most successful DRI's implemented in Social Science in terms of Skills, Systems and Support based initiatives. As these projects are currently unpublished research they have been consciously obfuscated and described at a high level.

\subsection{Skills-Based Initiatives}

The following interventions were focused on demonstrating the value of how advanced digital skills can lead to new research opportunities. In both instances, new skills were made available to researchers through targeted collaborations with digital experts.

Agent Based Modelling. A group of Geographers had built a large longitudinal dataset over a 30-year period and carried out typical statistical analysis on the data. Given the size and content of the dataset the researchers were not realizing the full potential of their data. An opportunity was identified to apply a new research method and analytical technique, which was outside the skillset of the researchers. A specialist research software engineer was injected into the team and developed a prototype agent-based model 
that was grounded in the data. This model was then run using our institution's highperformance computer, creating new data and insights for the researchers to work with. This exposure to new digital possibilities led to an increase in their research ambition.

Virtual Reality. Digital technologies can be extremely effective at engaging people in ideas, particularly in immersive experiences. A specialist virtual reality (VR) expert was sought to collaborate with Politics and Law researchers on developing an engaging experience. A portable VR game was developed from the ground-up with the intent of exposing players to the sensitive topics in an interactive and playful way. The system was designed to present players with different introductory instructions (independent variable) and capture in-game behaviours and decisions (dependent variable). Based on the players actions, a set of tailored outcomes are automatically generated and used to stimulate post-experience discussions. By using an immersive game, players will be able to better reflect on their experiences and perceptions, creating higher quality interview data.

\subsection{System-Based Initiatives}

The following interventions were designed to introduce new technologies for capturing, analysing and showcasing research data. Primarily these off-the-shelf and custom systems enhance the management of text, audio and image-based research data.

Optical Character Recognition. Many researchers work with paper-based research data, including historical records, newspapers and legislation. The challenge for researchers is the speed at which this data can be searched through, and the ability to hold an accurate mental model of the entire contents. Furthermore, the data is not easily shareable, and is at risk of accidental loss. Optical character recognition (OCR) essentially makes any text machine readable and the researchers were not aware of this possibility. By using a scanner and a text analytics tool, researchers were able to not only accurately search through the data, but also create statistical information a linguistic analysis. This transformed the nature of the research, with the ability to query the data in new and unprecedented ways.

Handwriting Technologies. The most widely used form of paper-based research data is in the form of hand written notes. These are made by field-based researchers making observations or notes around interviews. Sharing, searching and securing these notes can be a challenge, particularly given the amount that can be generated through ethnography. In recognition of this, a range of off the shelf 'smart pen' devices were trialled with researchers. These devices create digital copies of hand written notes using real paper and ink. Unique codes are printed onto each page, which allows the user to retroactively change the content of pages physically and digitally. Other than charging the device, there is no change to the practice of taking notes, meaning all the digital benefits are achieved with no barriers. The integrity of the data is protected and becomes easily 
searchable and shareable. The technology can even record audio, pairing digital notes with the audio files.

Automated Transcription. Researchers typically spend time transcribing their audio interviews themselves or pay for a transcription service to transcribe the data. The consequence of these resource (time/cost) heavy tasks is that the transcription of audio files is done on a priority basis. This means significant amounts of research data are not being fully utilized or shared; essentially the information is lost. Using Azure cognitive services and working with a software developer, a customized solution was developed that allows researchers to save audio files to their computer and automatically return a transcript. This can be done in bulk, meaning all existing audio files can be transcribed and even translated automatically. This then creates the opportunity for researchers to effectively search through their audio data and even conduct quantitative analysis.

\subsection{Support-Based Initiatives}

The following interventions were intended to explore the role of new specialized support services. Their main aim is to offer consultancy to researchers on the effective use of digital tools and technologies for their research.

Research Software Engineering. Researchers in Geography who conduct computationally intensive research were not aware of nor able to realise the full potential of their work due to the perceived technical barriers. An expert software engineer was introduced into the research team, and worked with the researchers on restructuring their code and data, which in turn allowed them to fully utilise the high performance computing facility at our institution. During the project, the researchers were also exposed to the potential of cloud computing. The initiative meant that the researchers could not only complete what they originally planned faster, but could run more simulations with the same resources. Optimisation of their code meant that they are now able to run larger models, increasing the accuracy and scale of their outputs i.e. they can model entire countries rather than a few islands.

Research Data Management. In May 2018 the GDPR [11] becomes enforceable, and will strengthen the rights of users over their data. Social Science is one of the research areas to be strongly affected by this change, as much of the work involves the digital capture and management of personal data. However, researchers were generally unaware of the new regulations. In response to this, a technology-legal expert was consulted to review the current governance processes around personal data how data is shared. Working with researchers, the legal expert amended and reinforced the institutions existing data access and user agreements in full recognition of the legislation and digital technologies used by researchers. This intervention will allow researchers to continue to digitally capture personally identifiable data in an ethical and legal way. 


\section{$5 \quad$ Digital Research Initiatives Framework}

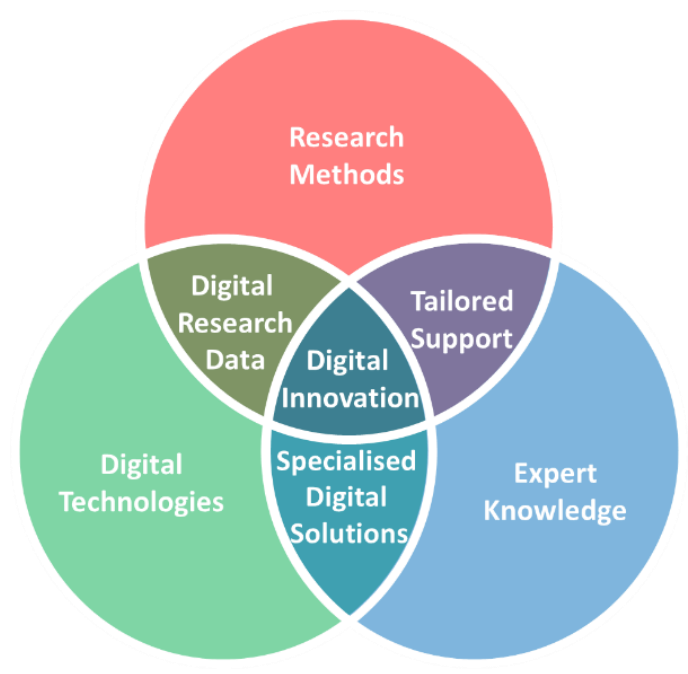

Fig. 1. The Digital Research Initiatives Framework (DRIF) allows institutions to assess current digital research capabilities and assist in the design of digital initiatives.

Following the implementation of the DRI's in the faculty, we began to reflect on the areas of success. While the initial focus of the DRI's was on skills, systems and support, it became clear through the implementation that there were attributes that were more impactful than others. This led to the formation of the DRI Framework (Fig.1) which is based on the relationship between three specific types of intervention in an academic setting. The first are Digital Technologies which are broadly defined as systems (hardware and/or software) that create or manipulate digital data. The second are Research Methods which are defined as systematic processes for creating data or concepts with the intent of answering a scientific research question. The third is Expert Knowledge, which refers to specific expertise in an area of specialism. We now consider the areas of intersection between these characteristics and relate back to the initiatives above.

Digital Research Data. Digital technologies are often know to create or capture digital data. However, is only when they are used as a part of a specific research method that digital research data is generated. Consider both OCR and handwriting technologies, these produced digital research data only when used as a part of the research process.

Specialised Digital Solutions. Digital technologies are ubiquitous in academic institutions and industry. However, simply having access to a technology does not make it effective or useful. Experts with specialist knowledge can help to customise, build and advise on the best use of the tools for a given purpose. Even without designing a specific technology from the ground up in a research context, these specialized solutions can be of use to researchers. The automated transcription is a clear example of this, where the 
digital tools were available and expert knowledge helped to construct a service around the technology - all of which was driven without a specific research method in mind.

Tailored Support. While researchers are experts in their specific area of interests, there are techniques and principles from other fields, particularly in Computer Science, that can advance their research. This was demonstrated by the introduction of the research software engineer in use of parallelization and the lawyer in managing research data.

Digital Innovation. Digital Innovation is where digital technologies, research methods and expert advice come together. This is the goal of our institution's digital research strategy, to maximize the interplay of these three areas. The immersive VR environments and agent based modelling projects were the most revolutionary for the researchers involved, as it opened new avenues and increased the ambitions of their research.

\subsection{Using the Digital Research Initiative Framework}

The framework can be used to assess the readiness of an institution for digital research transformation, but also to help guide the types of areas that might be implemented. The aim is to position a project or opportunity in the framework based on the three attributes, and to then determine the type of intervention required to 'pull' the project toward the centre. The first step is to assess an institution's capabilities in terms of three areas, research methods, digital technologies and expert knowledge. The second is to consider how these areas currently, or could, overlap to create new opportunities, these can often be the low hanging fruit. The final step is to consider how areas of overlap might be further enhanced, and 'pulled' toward the centre of the framework by introducing the appropriate third attribute. For example, our institution has used the framework to design three new exploratory and transformational projects

- Internet of Things devices (digital technologies) are built by non-researchers (expert knowledge). By considering the role of research methods, a new initiative was identified to explore the ethical considerations for use of this technology in research.

- A Sociology researcher is using a linguistic analytical tool (digital technologies) to apply a specialist technique (research method) to digital text. By considering the role of expert knowledge, a new initiative was identified to introduce a machine learning expert to help further develop the technique.

- Specialist economics researchers are developing code (expert knowledge) and exploring game theory (research method). By considering digital technologies, a new initiative was identified to introduce an eye-tracking device to capture new data.

To the best of our knowledge there are no other examples in the literature of conceptual frameworks for assessing the digital readiness of an academic institution. Although notably Arribas et al., [12] have proposed such a readiness framework in the context of the fashion industry. However their framework is exclusively grounded in digital technologies and drivers, and unlike the DRIF, fails to consider the role of expert 
knowledge. In a broader context, the DRIF directly informs Matt et al., [13] Digital Transformation Framework by proposing a series of new elements to be included in each of their "four cornerstones" of digital transformation; thereby grounding it in the context of an academic research institution.

\section{Conclusion}

Universities are complex organisations that operate in a challenging climate. It can therefore be arduous for institutions to define areas for transformation and successfully implement meaningful change. The strategic framework presented here enables institutions to introduce digital transformation in research via a three-pronged approach. By assessing the state of research methods, digital technologies and expert support available to researchers, this framework was used to successfully deploy practical methods for achieving our institution's digital research strategy.

\section{References}

1. Altbach, P.G., Reisberg, L. and Rumbley, L.E., (2009) Trends in Global Higher Education : Tracking an Academic Revolution. UNESCO 2009 World Conference on Higher Education 278 . doi: 10.1016/j.bse.2004.04.006

2. Gewirtz, S. and Cribb, A., (2013) Representing 30 years of higher education change: UK universities and the Times Higher. Journal of Educational Administration and History 45:58-83. doi: 10.1080/00220620.2013.730505

3. Research Councils UK, Hefce, Universities UK, Wellcome (2016) Concordat on Open Research Data

4. Chassang, G., (2017) The impact of the EU general data protection regulation on scientific research. E-Cancer Medical Science 11:1-12 . doi: 10.3332/ecancer.2017.709

5. UK RC Global Challenges Research Fund. https://www.ukri.org/research/global-challenges-research-fund/. Accessed 17 Apr 2018

6. Davé, A., Hopkins, M., Hutton, J., Krčál, A., Kolarz, P., Martin, B., Nielsen, K., Rafols, I., Rotolo, D., et al., (2016). Landscape review of interdisciplinary research in the UK.

7. Brynjolfsson, E., and McAfee, E., (2014) The second machine age: Work, progress, and prosperity in a time of brilliant technologies, 1st ed. W.W. Norton \& Company Ltd.

8. Wakeham Review (2016) Wakeham Review of STEM Degree Provision and Graduate Employability. 90

9. Van Der Wende, M., International academic mobility: towards a concentration of the minds in Europe. European review 23.S1 (2015): S70-S88.

10. Stilgoe, J., Lock. S.J., and Wilsdon J., (2014) Why should we promote public engagement with science? Public Understanding of Science 23:4-15 . doi: 10.1177/0963662513518154

11. European Parliament (2016) General Data Protection Regulation 2016/679. EUR Lex 11:1341 .doi: http://eur-lex.europa.eu/pri/en/oj/dat/2003/1_285/1_28520031101en00330037.pdf

12. Arribas, V., and José, A., (2016). Digital transformation of a small fashion house: a PLM implementation. Global Fashion Conference 2016, Stockholm

13. Matt, C., Hess, T., and Belian, A., (2015) Digital transformation strategies. Business \& Information Systems Engineering 57.5 (2015): 339-343. 